médecine/sciences $1989 ; 5: 138-144$

\title{
L'infection HIV et la transfusion sanguine
}

Le dépistage systématique des dons de sang séropositifs contre le virus de l'immunodéficience humaine (HIV), mis en place le $1^{\text {er }}$ août 1985, révèle une diminution de la prévalence de la séropositivité au niveau des donneurs de sang, en France comme aux États-Unis. Les éléments de dépistage dans les centres de transfusion sont un entretien médical avant chaque don, afin d'exclure les donneurs présentant un facteur de risque d'infection HIV, et un test sérologique (Elisa) mettant en évidence les anticorps dirigés contre le virus. Cependant, ce test ne permet pas de détecter les sujets qui, récemment infectés, sont encore séronégatifs. Les études sérologiques portant sur les malades transfusés après le $\mathrm{l}^{\mathrm{er}}$ août 1985 vont permettre d'apprécier le risque transfusionnel résiduel actuel d'infection HIV.

\section{Jean-Jacques Lefrère Charles Salmon}

\section{RÉFÉRENCES}

1. Andreani T, Modigliani R, Le Charpentier $Y$, et al. Acquired immunodeficiency with intestinal cryptosporidiosis: possible transmission by haitian whole blood. Lancet 1983 . $1: 1187-91$.

2. Ward JW, Deppe DA, Samson S, et al. Risk of human immunodeficiency virus infection from blood donors who later developed the acquired imunodeficiency syndrome. Ann Intern Med 1987; 106 (1): 61-2.

3. Reydiboym M, Laporte A, Brunet JB. Étude des cas de SIDA d'origine présumée transfusionnelle. Bull Epidemiol Hebd 1988; 22 : 85-6.

\section{ADRESSE}

J.-J. Lefrère: chef de service de l'unité de recherches cliniques de l'INTS. Ch. Salmon: directeur général de l'INTS. Institut national de transfusion sanguine, 6 , rue Alexandre- a transfusion sanguine a joué, dans la dernière décennie, un rôle néfaste dans la dissémination dans le monde du virus de l'immunodéficience humaine (human immunodeficiency virus) (HIV). En France, près de 6,5\% des cas de syndrome d'immunodéficience acquis (SIDA) présentent ou ont présenté comme seul facteur de risque d'infection HIV une transfusion sanguine reçue entre 1978 et juillet 1985 . Le premier cas mondial de SIDA post-transfusionnel a été rapporté en janvier 1982 chez un hémophile. Le premier cas français, survenu à la suite d'une transfusion pratiquée à Haïti, a été décrit en 1983 [1]. Cette forme de transmission de l'infection HIV et le rapprochement que l'on pouvait faire avec l'épidémiologie de l'hépatite $B$ ont été d'importants arguments en faveur de l'origine virale du SIDA.

Comme le virus HIV est présent au niveau des éléments figurés et du plasma, la plupart des dérivés sanguins, qu'il s'agisse du sang total, des concentrés globulaires, plaquettaires, leucocytaires, du plasma frais congelé, des concentrés en facteurs de coagulation, ont pu contaminer des receveurs, et tout sujet transfusé était, avant la mise en place du dépistage des dons séropositifs, sujet à risque. Seules l'albumine et les immunoglo- bulines, en raison d'un mode de préparation qui fait intervenir l'alcool, n'ont pas transmis l'infection. Les études séro-épidémiologiques n'ont révélé aucun cas d'infection HIV après utilisation d'immunoglobulines humaines, bien qu'il ait été rapporté des exemples de séropositivité HIV chez des receveurs d'immunoglobulines (il s'agissait d'un transfert passif d'immunoglobulines antiHIV provenant du donneur et disparaissant en quelques mois chez le receveur).

\section{L'infection HIV transfusionnelle}

Avant la mise en place du dépistage systématique des dons de sang ( $\mathrm{l}^{\mathrm{er}}$ août 1985), rendue possible par la découverte du virus et la mise au point de tests de dépistage, certains receveurs ont été contaminés par le sang de donneurs infectés. Ces donneurs étaient parfaitement asymptomatiques, comme la majorité des sujets porteurs du virus, et appartenaient à des populations reconnues ultérieurement à risque d'infection HIV.

La transfusion de sang infecté par le virus fut-elle constamment contagieuse? Si toutes les études s'accordent à reconnaître que la transfusion de sang contaminé est hautement infectante, certains auteurs ont sug- 
géré que le risque ne serait pas absolument de $100 \%$ [2]. Il convient cependant de souligner la difficulté pratique de ces études rétrospectives (dans plus de la moitié des cas, la recherche du donneur ou même du numéro des poches des produits sanguins n'aboutit pas), et l'on peut se demander si les receveurs non contaminés ne l'avaient tout simplement pas été parce que les donneurs n'étaient pas encore touchés, lors du don, par l'infection HIV.

Les enquêtes épidémiologiques [3] ont montré que la moyenne d'âge des SIDA transfusionnels était nettement supérieure à celle des autres facteurs de risque : près de $60 \%$ des cas transfusionnels ont plus de 50 ans, alors que moins de $13 \%$ de l'ensemble des cas de SIDA est dans cette tranche d'âge. Le sex ratio (hommes. /femmes) des malades infectés par transfusion a le chiffre le plus près de 1 en comparaison des autres groupes à risque [3, 4].

En raison de la quantité importante de virus inoculé, on a pensé que la période d'incubation d'un SIDA transfusionnel pouvait être plus brève que celle du SIDA lié à un contage sexuel. L'infection transfusionnelle réalise en effet un apport massif et unique de HIV par voie veineuse; cependant l'infection par voie sexuelle peut occasionner des apports faibles mais répétés de virus. En réalité, la durée exacte de l'incubation du SIDA post-transfusionnel n'est pas encore connue avec exactitude, mais pourra être établie par le suivi des sujets séropositifs infectés par transfusion, dont la date de contamination est connue avec précision. Les premières estimations, à l'aide de modèles mathématiques [5], donnent une incubation moyenne de 54 mois (4,5 ans), avec sept ans de recul sur les premiers cas. Toutefois, il convient de tenir compte du fait qu'un nombre important de malades transfusés avec du sang infecté par le HIV sont décédés de la maladie qui avait nécessité une transfusion, avant d'avoir eu le temps de développer un SIDA : par ailleurs, les cas à incubation longue n'ayant pas encore été diagnostiqués, ce chiffre est sûrement une sous-estimation de la réalité.

Rien ne distingue le SIDA transfusionnel des SIDA d'origine véné$\mathrm{m} / \mathrm{s} n^{\circ} 3$ vol. 5 , mars 89 rienne ou toxicomane, et le syndrome lymphadénopathique (stade III de la classification du center for disease control, CDC) peut également être observé. Les sujets infectés par transfusion peuvent à leur tour contaminer, par voie sexuelle, leur partenaire : environ $10 \%$ des épouses des hémophiles infectés par transfusion ont été trouvées séropositives; le risque semble plus faible (mais existe indéniablement) dans le sens femme $\rightarrow$ homme : une enquête sérologique récente [6] a porté sur les époux de femmes contaminées par transfusion avant le dépistage des dons séropositifs : sur 47 maris ayant eu au moins un an de rapports non protégés avec leur femme, un a été trouvé séropositif, sans avoir d'autre facteur de risque d'infection HIV.

Le nombre de SIDA dus à des transfusions contaminantes effectuées avant le dépistage systématique des dons séropositifs restera sans doute élevé durant quelques années, à cause de la durée d'incubation de la maladie. Par la suite, en raison de l'application des mesures préventives, le nombre de SIDA d'origine transfusionnelle devrait diminuer avec le temps. Il est donc difficile de prévoir le nombre exact de SIDA post-transfusionnels dans les prochaines années, mais on peut penser que ce nombre peut augmenter, pendant quelques années encore, parallèlement à celui des SIDA d'autres origines.

\section{Les éléments du dépistage dans les centres de transfusion}

- Un entretien médical avant chaque don vise à exclure les donneurs présentant un facteur de risque d'infection HIV. Les établissements de transfusion sanguine recommandent en effet aux volontaires de s'abstenir de donner leur sang s'ils appartiennent aux groupes exposés. Les sujets reçoivent alors les renseignements leur permettant de se rendre dans un centre de dépistage, où ils peuvent bénéficier d'un test sérologique. Les populations à risque sont actuellement les suivantes: sujets homosexuels ou bisexuels, sujets toxicomanes ou anciens toxicomanes par voie veineuse, sujets hétérosexuels à partenaires multiples, sujets origi- naires d'un pays à risque (Afrique), sujets transfusés, essentiellement avant le $1^{\text {er }}$ août 1985 , partenaires hétérosexuels des précédents.

Une enquête épidémiologique française récente a révélé que $52 \%$ des sujets interrogés pensaient que le don de sang faisait encourir un risque de contamination par le HIV (le matériel utilisé pour le prélèvement du sang du don est naturellement stérile et à usage unique) : ce chiffre met l'accent sur la nécessité de poursuivre les campagnes d'information de la population générale.

- Depuis le 1er août 1985, le dépistage des anticorps anti-HIV est obligatoire en France pour tous les dons de sang (arrêté du 23 juillet 1985, abrogé et remplacé par l'arrêté du 3 novembre 1986): ce dépistage est effectué à l'aide d'un test immunoenzymatique (Elisa), dont la positivité doit être, en raison de la possibilité de faux positifs [7,8], confirmée par un test de western-blot* (figure 1, p. 140). Tout prélèvement positif est exclu de l'utilisation transfusionnelle. Après confirmation de la séroposivité par western-blot, le donneur est convoqué et informé par un médecin de l'établissement de transfusion sanguine.

- L'inactivation virale des concentrés de facteurs de la coagulation (par chauffage puis par solvants détergents $^{* *}$ ) a permis d'assurer une sécurité maximale au traitement transfusionnel des hémophiles. Ces concentrés sont mis au point à partir du plasma d'un grand nombre de donneurs, et la présence d'un seul

\footnotetext{
* Western-blot = immunoblot: voir la légende de la figure 1, page 140.

* Le procédé solvant détergent a été mis au point par le New York Blood Center, dans le but d'inactiver non seulement le HIV, mais aussi les virus $B$ et non- $A$, non- $B$ des hépatites. Depuis l'emploi des solvants détergents, aucun cas de séroconversion HIV n'a été rapporté chez un hémophile transfusé avec un concentré anti-hémophilique ainsi inactivé sur le plan viral.
} 
Figure 1. Le western-blot (ou immunoblot) identifie les différents types d'anticorps sériques antiHIV du sujot. Principe du test : (a) les différentes protéines du virus sont dénaturées et séparées selon leur poids moléculaire par électrophorèse sur gel de polyacrylamide; (b) puis elles sont transférées sur une membrane de nitrocellulose qui reproduit les migrations des protéines. Cette membrane est ensuite découpée en bandes: chaque bande est incubée avec le sérum à tester; (c) les anticorps anti-HIV se fixent aux protéines virales qui leur correspondent. Puis une réaction immunoenzymatique les révèle (apparition d'une bande colorée). II faut, pour affirmer une séropositivité HN, la présence d'au moins deux anticorps différents, dont un doit être un anticorps antienveloppe. Interprétation do l'illustration (document fourni par A.-M. Couroucé): (1) Aspect typique d'infection HN-1 (présence de tous les anticorps). (2) Sujet séropositif HN-1, avec disparition des anticorps anti-core (c'est-à-dire dirigée contre un antigène du core viral) (signe d'évolutivité). (3) et (4) Aspect de début de séroconversion. (5) et (6) Réaction non spécifique d'une infection HIV-1 lanticorps anti-core isolé, sans anticorps anti-enveloppe). Les bandes correspondant à l'enveloppe sont les GP 160, GP 110 et GP 41. Les bandes correspondant au core viral sont les P55, P25 et $P 18$ (dérivés de la protéine Gag).

Figure 2. Lo tost Elisa HIV (enzymo linked immunosorbent assay). Ce test révèle la présence dans le sérum d'anticorps anti-HIV, sans distinguer les différents types d'anticorps. Comme ce test a des " faux positifs », la positivité de l'Elisa doit être confirmée par un western-blot. II existe deux types de test Elisa: (1) Elisa de "première génération » : le sérum à tester est (après dilution) mis en contact avec l'antigène viral absorbé sur phase solide (particules virales purifiées puis lysées obtenues à partir de virus produits in vitro par des lignées de cellules infectées par le HIV). Les anticorps anti-HIV se fixent sur les antigènes viraux: l'addition d'une antiglobuline marquée à la peroxydase, puis du substrat de cette enzyme, provoque une réaction colorée. (2) Elisa de " deuxième génération »: même principe, mais avec des protéines virales de recombinaison ou de synthèse. La spécificité est meilleure qu'avec les Elisa de
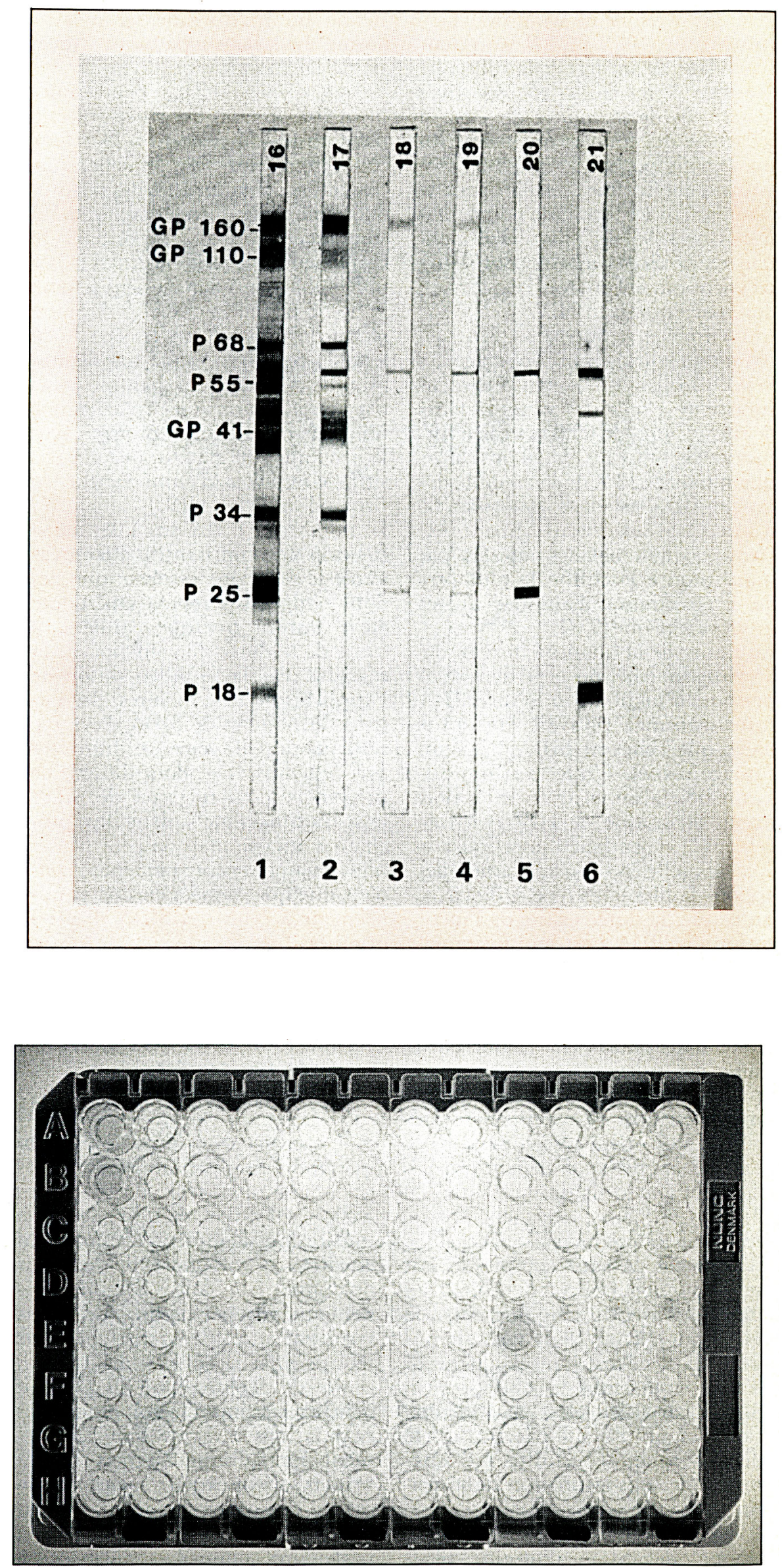


\begin{tabular}{|c|c|c|c|c|}
\hline \multicolumn{5}{|c|}{$\begin{array}{c}\text { Tableau I } \\
\text { PRÉVALENCE DES DONS DE SANG SEROPOSITIFS } \\
\text { AU CENTRE NATIONAL DE TRANSFUSION SANGUINE } \\
\text { ENTRE AOUTT 1985 ET JUIN } 1988^{*}\end{array}$} \\
\hline & $\begin{array}{c}1985 \\
\left(2^{\mathrm{e}} \text { semestre }\right)\end{array}$ & 1986 & 1987 & $\begin{array}{c}1988 \\
\left(1^{\text {er }} \text { semestre) }\right.\end{array}$ \\
\hline Nombre de dons & 110683 & 236897 & 237351 & 101697 \\
\hline Nombre de dons séropositifs & 102 & 176 & 102 & 21 \\
\hline Taux de séropositivité (\%/oo) & 0,92 & 0,74 & 0,42 & 0,20 \\
\hline
\end{tabular}

* Données communiquées par le Dr D. Richard.

porteur du virus peut suffire à contaminer le concentré. Ces moyens d'inactivation virale, qui enlèvent à un éventuel virus son pouvoir infectieux et son pouvoir immunogène, sont malheureusement inutilisables pour d'autres dérivés sanguins.

\section{Prévalence de la séropositivité au niveau des dons de sang}

Le risque de contamination transfusionnelle par concentré globulaire avant la mise en place du dépistage systématique des dons de sang séropositifs était lié d'une part au nombre de sujets séropositifs parmi les donneurs de sang de cette époque, d'autre part au nombre d'unités transfusées au malade.

Le taux de séropositivité des donneurs de sang en France a régulièrement diminué depuis la mise en place du dépistage systématique.

- Sur les cinq mois de 1985: 990 dons furent trouvés séropositifs parmi 1553287 testés (soit 0,64 pour 1 000). Le taux de séropositivité variait de façon importante avec les différentes régions de France : il était à 0,92 pour 1000 pour les donneurs du Centre national de transfusion sanguine, c'est-à-dire de Paris et d'une partie de la région parisienne (Tableau I).

- En 1986: 1694 dons séropositifs parmi 4085658 dons testés $(0,41$ pour 1000 ).

- En 1987 : 1278 dons séropositifs parmi 4203316 dons testés $(0,30$ pour 1 000).

- En 1988 : le chiffre est de l'ordre de 0,20 pour 1000 . déjà donné leur sang dans le centre de transfusion (le taux passe chez eux de 0,23 pour 1000 au ler $^{\text {er }}$ trimestre 1986 à 0,09 pour 1000 fin 1987); la diminution est moins nette pour les «nouveaux donneurs » (inconnus du centre de transfusion), souvent recrutés à partir d'unités mobiles (camions stationnant dans les rues): près de $70 \%$ des dons trouvés séropositifs proviennent en effet de «nouveaux donneurs», alors que ceux-ci représentent environ $15 \%$ des donneurs. En raison de cette différence parmi ces deux types de donneurs, la diminution de la prévalence globale de la séropositivité des dons de sang pourrait être accrue, comme cela a été récemment envisagé, par l'abandon, pour certaines régions à haute incidence de séropositivité, des collectes de sang sur la voie publique.

\section{Épidémiologie des donneurs} de l'entretien médical qui précède le don, ainsi que les conseils donnés à la population générale à l'occasion de différents appels publics incitant les sujets à risque à ne plus utiliser le don de sang comme test diagnostique. Enfin, l'ouverture de centres de dépistage, à Paris puis dans l'ensemble des départements français, a permis à tout volontaire d'être testé de manière gratuite, anonyme et, si besoin, répétée.

Cette diminution de la prévalence des dons de sang séropositifs est surtout nette pour les «donneurs connus », c'est-à-dire les sujets ayant

L'interrogatoire et le suivi des sujets reconnus séropositifs à l'occasion d'un don de sang révèle que la majorité d'entre eux, en 1985 comme en 1988 , appartient aux populations à risque [8, 9] (Tableau II). Sur le plan régional, on trouve, par ordre de taux décroissant de séropositivité parmi les donneurs de sang, les départements d'outre-mer, Paris et la région parisienne, la Provence-Côted'Azur et le Languedoc-Roussillon. Il serait cependant abusif, surtout depuis la mise en place des mesures

\begin{tabular}{|l|c|c|c|c|}
\hline \multicolumn{5}{|c|}{$\begin{array}{l}\text { Tableau II } \\
\text { ÀCTEURS DE RISOUE DE 286 SUJETS DETECTÉS } \\
\text { DU CENASION D'UN DON DE SANG DANS LE CADRE }\end{array}$} \\
\hline $\begin{array}{l}\text { Sujets } \\
\text { détectés }\end{array}$ & $\begin{array}{c}1985 \\
\text { (2e semestre) }\end{array}$ & 1986 & 1987 & $\begin{array}{c}1988 \\
\text { (1er semestre) }\end{array}$ \\
\hline Homosexuels & 54 & 73 & 40 & 6 \\
\hline Toxicomanes & 11 & 21 & 13 & 2 \\
\hline $\begin{array}{l}\text { Partenaires hétérosexuels } \\
\text { de sujets à risque }\end{array}$ & 9 & 13 & 5 & 4 \\
\hline $\begin{array}{l}\text { Transfusés avant } \\
\text { le 1er août 1985 }\end{array}$ & 1 & 3 & 1 & 0 \\
\hline Origine africaine & 0 & 2 & 2 & 0 \\
\hline $\begin{array}{l}\text { Sans facteur } \\
\text { de risque classique }\end{array}$ & 6 & 12 & 7 & 1 \\
\hline
\end{tabular}




\section{RÉFÉRENCES}

4. Curran JW, Lawrence DN, Jaffe $\mathrm{H}$, et al. Acquired immunodeficiency syndrome (AIDS) associated with transfusions. $N$ Engl J Med 1984 ; 310 : 69-81.

5. Lui KJ. A model based approach for estimating the mean incubation period of transfusion associated acquired immunodeficiency syndrome. Proc Natl Acad Sci USA $1986 ; 8$ : 3051-5.

6. Lefrère JJ, Vittecoq $\mathrm{D}$, North ML, et al. Risk of female-to-male transmission of HIV from women infected by transfusion. AIDS $1988 ; 2$ : 137-8.

7. Couroucé AM, Muller JY, Richard D. False positive western-blot reactions to human immunodeficiency virus in blood donors. Lancet $1986 ; 2$ : 921-2.

8. Lefrère JJ, Couroucé AM, Lucotte G, et al. Follow-up of subjects with isolated and persistent anti-core (anti-p24 or anti-pl7) antibodies to HIV. AIDS 1988 ; 2 : 287-90.

9. Lefrère JJ, Muller JY, Richard D, Muller A, Garetta M, Salmon C. HIV seropositivity and blood donations in Paris 1985-1987. Vox Sang 1988 ; 54 : 123-4.

10. Lefrère JJ, Richard D, Couroucé AM, et al. Risk factors of HIV seropositive subjects detected through blood donations in France 1985-1988. Transfusion (sous presse).

11. Ranki AM, Krohn M, Allain JP, et al. Long latency precedes over seroconversion in sexually transmitted human immunodeficiency infections. Lancet $1987 ; 2$ : 589.

12. Groopman JE, Caiazzo T, Thomas MA, et al. Iack of evidence of prolonged immunodeficiency virus infection before antibody seroconversion. Blood 1988 ; 71 (6) : 1752-4.

13. Couroucé AM. I_atency preceding seroconversion in sexually transmitted HIV infection. Lancet 1987 ; 2 : 1025.

14. Kwok S, Mack DH, Mullis KB, et al. Identification of human-immunodeficiency virus sequences by using in vitro enzymatic amplification and oligomer cleavage detection. J Virol 1987 ; 61 : 690-4

15. Ou CY, Kwock S, Mitchell SW, et al. DNA amplification for direct detection of HIV-I in DNA of peripheral blood mononuclear cells. Science 1988 ; 239 : 295-7.

16. Ward JW, Holmberg SD, Allen JR, et al. Transmission of human immunodeficiency virus (HIV) by blood transfusions screened as negative for HIV antibody. N Engl J Med 1988 ; $318: 473-8$.

17. Donegan E, Johnson D, Remedios V, Cohen S. Mass notification of transfusion recipients. IVth International Conference on AIDS. Stockholm, 1988 ; 2 (353) : 7713 A.

18. Caiazza SS. Alternative sites for screening blood for antibodies to Aids virus. $N$ Engl J d'exclusion des donneurs à risque, de voir dans ces données un reflet de la population générale. En effet, bien que les sujets à risque aient été très tôt invités à ne pas donner leur sang, un certain nombre d'entre eux a recouru, faute d'existence de centres de dépistage gratuit et anonyme, au don du sang comme test diagnostique. Dans notre expérience, plus de $90 \%$ des sujets reconnus séropositifs par don de sang dans le cadre du Centre national de transfusion sanguine appartenaient, de 1985 à 1988, aux populations à risque classique et avaient donné leur sang pour avoir le test.

L'information et le suivi des sujets reconnus séropositifs à l'occasion d'un don de sang. Les donneurs dont le sang a été reconnu séropositif (avec confirmation par western-blot) reçoivent une lettre leur signalant qu'une anomalie biologique a été détectée lors de leur don et leur demandant de prendre contact avec la consultation médicale du centre de transfusion. Le sujet est informé de sa séropositivité et de ses conséquences: (a) l'abstention définitive du don de sang ; (b) la nécessité définitive de protéger les rapports sexuels par des préservatifs, car le sujet séropositif, potentiellement contaminant, peut infecter ses partenaires (ou se réinfecter s'ils sont euxmêmes séropositifs); (c) l'information de ses partenaires passés et actuels, afin qu'ils effectuent un test sérologique ; (d) la contre-indication de toute grossesse; (e) la nécessité d'un suivi clinique et biologique à une consultation spécialisée, afin de mettre en route une thérapeutique antivirale en cas de signes biologiques d'évolutivité annonçant une évolution irréversible et prochaine vers la maladie.

Certains dons de sang peuvent-ils échapper au dépistage actuel et être utilisés en transfusion ? Malgré le dépistage actuellement en cours au niveau de l'ensemble des centres de transfusion français, le risque de transmission transfusionnelle d'une infection HIV par un don séronégatif n'est pas entièrement nul, en particulier dans les régions où la prévalence de l'infection est élevée. Il y a à cela plusieurs explications :
- On ne peut éliminer la possibilité d'une erreur humaine à une des étapes du dépistage biologique, mais c'est un accident tout à fait exceptionnel.

- L'exclusion des sujets à risque lors de l'entretien médical qui précède le don peut avoir des failles, en raison de la qualité de l'interrogatoire (effectué dans des conditions souvent difficiles comme les collectes de rues ou le lieu de travail) ou de la sincérité des réponses des donneurs interrogés. Il est possible également que des sujets à risque puissent s'ignorer tels, comme une femme ayant eu des relations sexuelles avec un partenaire dont elle ignorait la bisexualité ou la toxicomanie. Or, si un sujet récemment infecté par le HIV donne son sang alors qu'il est encore virémique et non encore séropositif (c'est-à-dire entre le premier contact infectant avec le virus et l'apparition des premiers anticorps caractérisant la séroconversion), le dépistage actuel, basé sur la sérologie, ne repère pas le don : le test Elisa détecte les anticorps anti-HIV, non le virus lui-même. Cette phase de silence sérologique est de l'ordre de quelques semaines (inférieure à deux mois). Certains auteurs [11] ont suggéré que cette phase pourrait être parfois beaucoup plus longue, de l'ordre d'une année, mais cette donnée a été rapidement démentie [12, 13]. Le problème était d'importance, car un délai d'incubation aussi prolongé pouvait laisser supposer l'éclosion de nombreux nouveaux cas transfusionnels dans les prochaines années.

- Il a également été rapporté que des sujets pouvaient être infectés par le virus $\mathrm{HIV}$, inscrit sous forme d'ADN dans le génome de certaines cellules, sans que cet $\mathrm{ADN}$ provoque la synthèse des protéines virales, donc sans production de virions. Ces sujets n'exprimeraient pas d'anticorps anti-HIV : ils resteraient séronégatifs, bien qu'ils soient infectés (et peut-être infectants), et ne seraient pas reconnus par le dépistage biologique actuel, qui n'est fondé que sur la recherche d'anticorps anti-HIV. La technique de $\mathrm{PCR}^{*}$ (polymerase chain reaction), récemment mise au point [14, 15], permettrait sans doute

* Voir m/s no 8, vol. 4, p. 515. 


\begin{tabular}{|l|c|c|c|}
\hline \multicolumn{4}{|c|}{ Tableau III } \\
\multicolumn{4}{|c|}{$\begin{array}{c}\text { EVLLUTION DE LA CONSOMMATION DES PRODUITS SANGUINS } \\
\text { AU CENTRE DE TRANSFUSION DE L'HOPIAL FOCH* }\end{array}$} \\
\hline & 1986 (\%) & $1987(\%)$ & $1988(\%)$ \\
\hline Concentrés érythrocytaires & -5 & -13 & -13 \\
\hline Plasma frais congelé & +10 & -22 & -46 \\
\hline Albumine & +0 & +92 & +488 \\
\hline
\end{tabular}

* Hôpital de 1000 lits, avec une activité opératoire stable sur la période considérée. Les chiffres sont exprimés en pourcentage par rapport à l'année précédente. (Données communiquées par le Dr D. Vignon.)

de reconnaître ces sujets, mais elle est encore loin d'être standardisée et applicable en routine.

- Les études américaines et françaises ont estimé entre $1 / 40000$ à $1 / 1$ million $[9,16]$ le nombre de dons infectants et non repérés par le dépistage systématique fondé sur la mise en évidence des anticorps dirigés contre le HIV. Lors du dernier Congrès international de transfusion sanguine (Londres, juillet 1988), une étude américaine de la fréquence d'infection post-transfusionnelle HIV par du sang séronégatif estime ce chiffre entre $1 / 53000$ et l/357 000 .

Surveillance du risque d'infection transfusionnelle. Diverses études sont en cours chez les malades transfusés depuis le $1^{\text {er }}$ août 1985 ou chez des sujets polytransfusés sans autre risque d'infection HIV comme les enfants thalassémiques afin de rechercher une séropositivité développée après infection transfusionnelle. Cependant, un problème, soulevé par quelques auteurs lors du congrès de Stockholm de juin 1988, est l'ignorance où sont certains patients d'avoir été transfusés durant une intervention: dans une enquête américaine, $13 \%$ des malades ne savaient pas qu'ils avaient été transfusés [17].

Par ailleurs, des enquêtes portent actuellement sur les receveurs d'un don séronégatif provenant d'un sujet reconnu ultérieurement séropositif à l'occasion d'un nouveau don de sang. Ces enquêtes sont difficiles à mener, car il n'est pas aisé, dans un grand nombre de cas, de retrouver le receveur d'un produit sanguin donné. Ces études ont cependant per- mis de confirmer que le délai du silence sérologique précédant la séroconversion n'excédait pas six semaines.

De quels moyens pourrait-on disposer pour accroître la sécurité actuelle ? (1) L'antigénémie p25 (test antigène) détecterait sans doute un certain nombre de sangs parmi ceux effectués entre le premier contact infectant du virus et la séroconversion. Toutefois, des raisons de coût et de rentabilité ont fait différer jusqu'à présent son utilisation au niveau de tous les dons de sang. (2) La mise en évidence d'anticorps dirigés contre la protéine NEF pourrait permettre d'identifier un sang infecté mais non reconnu par la sérologie actuellement utilisée. (3) $\mathrm{La}$ technique de PCR permet la détection directe du génome viral dans les cellules du sujet infecté. Cette technique d'amplification de l'ADN proviral peut déceler un nombre très faible de copies dans les lymphocytes. Elle est fondée sur l'amplification spécifique d'une séquence génomique virale par une réaction en chaîne de l'ADN-polymérase, pour augmenter le nombre de copies de ce fragment et les rendre détectables par hybridation avec une sonde spécifique d'ADN marquée. Cependant cette technique est encore relativedans l'immédiat, d'envisager une utilisation pour le dépistage en routine des dons infectés par le HIV.

Ces techniques posent ainsi des problèmes de faisabilité ou de coût. Dans ces conditions, il a paru plus efficace de jouer sur d'autres possibilités telles que : ment lourde, et il paraît difficile,
- L'autotransfusion (ou transfusion autologue): le receveur reçoit son propre sang, prélevé dans les semaines ou les mois précédents. Le risque d'infection virale est alors nul. Toutefois, l'autotransfusion n'est possible que pour les interventions programmables à l'avance.

- La limitation des indications transfusionnelles, en incitant les anesthésistes et les chirurgiens à ne transfuser qu'en cas d'absolue nécessité. L'usage de plasma frais congelé, en particulier, est médicalement injustifié dans un grand nombre de cas, et il est souvent possible de le remplacer par un produit comme l'albumine, dont la transfusion ne fait courir aucun risque infectieux (Tableau III).

- Le développement, dans un avenir proche, de produits industriels, comme le facteur VIII (anti-hémophilique A) produit par méthode génétique.

- L'amélioration du dépistage des donneurs et des dons séropositifs par: (a) le renforcement de la sécurité du dépistage sérologique actuel, en jouant sur la sensibilité et la spécificité des trousses et sur la limitation maximale des erreurs humaines; (b) l'amélioration de l'interrogatoire lors de l'entretien médical qui précède le don, notamment dans les collectes de rue et pour les «nouveaux donneurs», afin de renforcer l'exclusion des donneurs à risque. Cependant, l'extension de l'infection, lente mais certaine, en dehors des populations à risque «classique», peut rendre cet interrogatoire moins efficace. C'est pourtant le seul filtre actuel pour les dons échappant au dépistage sérologique; (c) la multiplication des centres de dépistage gratuit et anonyme pour que les sujets désireux de se faire tester n'aient plus à recourir au don de sang pour avoir le test sérologique de manière confidentielle [18].

Hémophilie et infection HIV. Les concentrés de facteurs de la coagulation utilisés dans le traitement des hémophilies A et B étant mis au point à partir du plasma de plusieurs centaines de donneurs, les hémophiles ont été infectés en grand nombre avant la mise en place des mesures préventives. Actuellement, près de $45 \%$ des hémophiles français 
sont séropositifs, ce chiffre étant plus élevé pour certains centres parisiens, et moins élevé dans certains centres de province. Paradoxalement, après avoir été les sujets transfusés les plus exposés à l'infection HIV, les hémophiles sont dorénavant les plus protégés du risque d'infection transfusionnelle rétrovirale. En effet, au dépistage systématique des dons de sang séropositifs, a été associée, pour les fractions anti-hémophiliques, l'inactivation virale par chauffage à $56^{\circ} \mathrm{C}$ puis par solvants détergents. Ces moyens d'inactivation paraissent d'une efficacité absolue: aucun cas n'a jusqu'à présent été rapporté d'hémophile infecté par des produits transfusionnels soumis à ces mesures préventives.

Comme avec les autres facteurs de risque de l'infection HIV, on observe, parmi les hémophiles polytransfusés, des sujets appelés «séronégatifs paradoxaux »: de tels sujets restent séronégatifs malgré le grand nombre de transfusions reçues avant le dépistage des dons de sang séropositifs. Ces sujets ont-ils des éléments de résistance individuelle contre le virus ? Sont-ils porteurs du virus ou de séquences virales en dépit de leur séronégativité persistante? La recherche d'anticorps anti-NEF, la technique de PCR, actuellement à l'étude dans ce contexte pourront peut-être permettre de résoudre tout ou partie de ce problème.

Quant à l'évolution des hémophiles séropositifs vers le SIDA, elle semble obéir aux mêmes règles que celle des autres facteurs de risque.

La transfusion en Afrique. La transfusion a été en Afrique un facteur de dissémination du virus. Elle le reste encore, étant donné l'absence de dépistage des dons séropositifs dans bon nombre de pays africains, dans lesquels la prévalence de la séropositivité est pourtant élevée plus que partout ailleurs dans le monde.

\section{Le HIV-2}

Le HIV-2 est, comme le HIV-l, transmissible par transfusion sanguine. Les tests immunoenzymatiques (Elisa) (figure 2, p. 140) HIV-1 donnent des résultats positifs avec les sérums des sujets infectés par le HIV- deux types de virus en raison des homologies au niveau du gène gag). Un western-blot spécifique du HIV2 peut ensuite affirmer le diagnostic. En raison d'une part de l'existence de ces réactions croisées, d'autre part de la rareté de l'infection HIV-2 en France, la demande en première intention d'une sérologie spécifique du HIV-2 n'est pour l'instant justifiée que si le sujet est originaire d'un pays à risque (Afrique de l'Ouest) ou a eu un partenaire originaire d'un pays à risque. Le dépistage systématique des dons de sang pour le HIV2 n'est donc pas pour l'instant envisagé, mais une surveillance épidémiologique est instituée.

D'autres virus HIV pourraient-ils émerger, dans les prochaines années, en Afrique ou ailleurs, et se répandre dans le monde par l'intermédiaire, entre autres, de la transfusion? Il semble difficile d'exclure cette possibilité, quand on se souvient que le problème du SIDA s'est révélé comme un coup d'éclair dans un ciel transfusionnel presque serein. La meilleure prévention de cet accident est la limitation de la transfusion à ses indications indispensables. Les transfusions dites « de confort » sont désormais proscrites.

\section{Conclusion}

Les spécialistes français et américains s'accordent à reconnaître que le risque transfusionnel actuel d'infection HIV est «acceptable». Il convient en effet de mettre en balance ce risque, devenu aujourd'hui tout à fait minime, et l'apport de la transfusion en médecine et en chirurgie, où des vies sont sauvées tous les jours grâce à elle.

Cependant, bien que le dépistage des dons séropositifs ait considérablement diminué le risque transfusionnel, les efforts doivent être poursuivis pour le réduire encore. A ce jour, les meilleures armes demeurent la connaissance, l'information et la prise de conscience à tous les niveaux

\section{Remerciements}

Nous voudrions remercier les docteurs D. Richard et D. Vignon ainsi que Madame A.-M. Couroucé

\section{Summary}

Human immunodeficiency virus infection and transfusion

The characteristics of human immunodeficiency virus (HIV) infection through the administration of blood products are given. The screening of HIV seropositive blood donations reveals a decrease of the seroprevalence of seropositivity between 1985 and 1988 in France as seen in the USA, due perhaps to a decline in repeat donors. The elements used to screen HIV-infected blood donors in transfusion centers are an interview before the blood donation and a serologic test (enzyme immunoassay) to identify specific antibodies. However, tests for HIV antibody do not detect all HIV-infected donors : some rare blood donations remain unscreened when performed before seroconversion. Control elements of the actual transfusionnal HIV infection risk are restrospective studies on the HIV serology of patients transfused since August lst 1985, date of the beginning of the systematic screening of blood donations.

\section{TIRÉS A PART}

J.-J. Lefrère 